\title{
Potential inaccuracy in recording the presence of diabetes in people with amputations of the lower limb in hospitals in England. Reply to Ahmed M, Moulik P and Macleod A [letter]
}

\author{
N. Holman • R. J. Young • W. J. Jeffcoate
}

Received: 3 September 2012 / Accepted: 13 September 2012 / Published online: 2 October 2012

(C) Springer-Verlag Berlin Heidelberg 2012

\section{Abbreviation \\ PCT Primary care trust}

To the Editor: We thank Ahmed et al for their valuable comment [1] and while we wish to counter one or two of their points, we are in general agreement with the warning highlighted by their letter.

The analysis in our paper [2] referred to the incidence of amputations among people with diabetes while the figures quoted in the letter relate to prevalence. The incidence of major amputation among people with diabetes for April 2007 to March 2010 in Shropshire County PCT and Telford and Wrekin PCT, which were included in the original paper, were 0.82 and 1.18 per 1,000 person-years, respectively. Further examination of the data shows that there were 30 major amputations in 28 patients in Shropshire PCT and 25 major amputations among 21 patients in Telford and Wrekin PCT in this time period. The analysis presented in our paper identified all amputations among patients registered with a GP belonging to a PCT irrespective of where the operation

\footnotetext{
N. Holman

Diabetes Health Intelligence,

Yorkshire and Humber Public Health Observatory,

York, UK

R. J. Young

Department of Diabetes and Endocrinology,

Salford Royal Hospital Foundation Trust,

Salford, UK

W. J. Jeffcoate $(\bowtie)$

Foot Ulcer Trials Unit, Department of Diabetes and

Endocrinology, Nottingham University Hospitals Trust,

City Hospital Campus,

Nottingham NG5 1PB, UK

e-mail: William.Jeffcoate@nuh.nhs.uk
}

was done. While the majority of the amputations in patients registered with GPs from these two PCTs occurred at Shropshire County and Telford and Wrekin NHS Trusts, a small number took place at other hospitals. Applying the same methodology adopted by Ahmed et al to our data yields results similar to those given in the letter [1] (prevalence of major amputations 0.68 per 1,000 people with diabetes [25 patients] for Shropshire County PCT and 0.89 per 1,000 people with diabetes [19 patients]) for Telford and Wrekin PCT. The authors highlight some patients with diabetes where the diagnosis was not recorded in discharge coding. Where this occurs, it will lead to an under-estimation of the scale of hospital use by patients with diabetes (rather than an over-estimation as suggested in the letter).

The potential limitations of data derived from hospital episode statistics (HES) are well recognised but, as argued in the paper [2], we believe that these limitations are now much less of a concern than they used to be, partly because of the financial incentive for hospital trusts in today's NHS to identify all people with diabetes, particularly surgical patients, and partly because of the close correspondence between the incidences we derived, both in terms of actual numbers and of geographical variation, and those published by the National Diabetes Audit (NDA), which uses completely different sources and analyses amputations occurring over the preceding 5 years. We spelled out the care with which each set of data must be interpreted in our paper [2].

The point about the weakness of assumptions deriving from the small numbers of amputations in each individual PCT is well made. Hitherto, the alternative to using data from the 151 PCTs in England has been limited to aggregations into the ten Strategic Health Authorities, but the use of very much bigger populations inevitably dampens the variation that can be documented. However, when the number of cases in the chosen areas is low it will be affected to a 
greater extent by the peaks and troughs that inevitably characterise the incidence of clinical events. In general, the use of 3 year moving means will help mitigate this effect.

And while we agree that data based on a national diabetes register (which is the eventual aim of the NDA) would be preferable, we do not agree that it is premature to compare PCTs in the way that we have done. The data must be interpreted with care but the demonstration of variation between PCTs is a wake-up call. The intention is not to point a finger of blame at individual clinical groups, but to trigger further work to determine why such apparent variation exists - both within countries and between them: work similar to that undertaken by Ahmed et al. We do, however, agree wholeheartedly about the danger of complacency that can be induced in areas in which a low reported incidence may be the result, at least in part, of poor coding practice. A similar sense of false security may also exist in conurbations in England that have a particularly high prevalence of Asians in their population. The main message to be derived from our work is that apparent variation between different health economies should remain under close scrutiny and reasons should be sought for its occurrence.

Contribution statement The authors were responsible for the conception, design and drafting of the manuscript, and approval of the final version for publication.

Duality of interest The authors declare that there is no duality of interest associated with this manuscript.

\section{References}

1. Ahmed M, Moulik P, Macleod A (2012) Potential inaccuracy in recording the presence of diabetes in people with amputations of the lower limb in hospitals in England. Diabetologia. doi:10.1007/ s00125-012-2735-6.

2. Holman N, Young RJ, Jeffcoate WJ (2012) Variation in the recorded incidence of amputation of the lower limb in England. Diabetologia 55:1919-1925 